\title{
Immunoglobulin G4-Related Mastitis: An Unusual Case of Recurrent Breast Abscess
}

\author{
Shruti BAJAD (1), Dhaval TANNA@, Gayatri EKBOTE (D), Muzaffar BINDROO (1), \\ Kanchan KAUR(D, Rajiva GUPTA (1)
}

Medanta-The Medicity, Rheumatology and Clinical Immunology, Gurugram, India

\begin{abstract}
Immunoglobulin G4-related mastitis (IgG4-RM) is an uncommon entity in clinical practice which has an evolving spectrum of manifestations and presently is of high clinical interest among rheumatologists. Since its closest differential remains breast carcinoma, the importance of describing this entity is in the fact that early diagnosis, awareness and timely management can save the patient from unnecessary surgical intervention and its complications. Tumefactive lesions are considered hallmark of this disease, but rarely abscess may also be the presenting feature. In this article, we describe a 24-year-old female patient of a very unusual case of IgG4-RM presenting as recurrent breast abscess, its successful management and discussion about novel treatment strategies.

Keywords: Breast abscess, immunoglobulin G4-related disease, immunoglobulin G4-related mastitis.
\end{abstract}

Immunoglobulin G4-related disease (IgG4-RD) is an immune-mediated fibroinflammatory disease. Previously described diseases such as Mikulicz's disease, Riedel's thyroiditis, and Küttner's tumor are now considered to be part of IgG4-RD spectrum. In 1995, a unique form of pancreatitis having autoimmune etiology and showing good response to steroids was described. ${ }^{1}$ Subsequently, these autoimmune pancreatitis (AIP) cases were found to be associated with elevated serum IgG4 concentration and unique histopathologic features. ${ }^{2}$ In recent times, IgG4-RD has been described in virtually every organ system: the biliary tree, pancreas, aorta, meninges, lymph nodes, salivary glands, periorbital tissue, breast, prostate, thyroid, pericardium and skin. ${ }^{1,3}$ Although tumefactive lesions are well described in the literature, very rarely, IgG4-RD may present as inflammatory pseudotumor or an abscess.
IgG4-related breast disease, better known as IgG4-related mastitis (IgG4-RM), presents with palpable breast lump and remains an important differential diagnosis of breast malignancy. Very few cases of IgG4-RM have been described in the literature. In this article, we describe a case of IgG4-RD presenting as recurrent breast abscess and its management.

\section{CASE REPORT}

A 24-year-old female patient with no known comorbidities developed sudden onset pain and redness in right breast. She was diagnosed with right-sided mastitis by primary care physician and treated with oral antibiotics and analgesics for a week. She had symptomatic improvement with treatment. Two months later, she again developed

Received: July 24, 2019 Accepted: August 17, 2019 Published online: November 27, 2019

Correspondence: Shruti Bajad, MD. Medanta-The Medicity, Rheumatology and Clinical Immunology, 122001 Gurugram, India. Tel: 9925076380 e-mail: shrutibajad@yahoo.in 
pain in right breast accompanied with ill defined erythematous swelling over right periareolar area and pus discharge from swelling and nipple. Ultrasonography of the breast showed loculated breast abscess of $5 \times 3 \mathrm{~cm}$. Fine needle aspiration cytology of the lesion showed sheets of pus cells without any evidence of dysplasia. She underwent incision and drainage of breast abscess and thick pus was drained. Pus cultures were sterile and gram stain and acid-fast bacilli (AFB) stain were normal. She was treated with antibiotics for two weeks. Her symptoms improved for a while but two weeks later she again developed swelling over right breast with pus and blood discharge from right nipple. She was brought to our hospital. On examination, she had right periareolar induration. Ultrasound of both breasts detected ill defined hypoechoic area measuring $50 \times 20 \mathrm{~mm}$ in right breast with hyperreflective surrounding parenchyma suggestive of surrounding inflammation (Figure 1). Her routine laboratory investigations were normal except mild leucocytosis and high erythrocyte sedimentation rate (ESR) (white blood cells: 11,400/cumm,
ESR: $55 \mathrm{~mm} /$ hour). Pus culture did not reveal growth of any micro-organism. True cut biopsy was performed which showed focal architectural distortion of breast tissue by dense inflammatory infiltrate of lymphocytes, plasma cells, histiocytes and foamy macrophages. There was dense sclerotic stroma and periductal inflammatory infiltrate (Figure 1). No granulomas were seen. AFB stain and special stains for fungus were negative. On immunohistochemistry, IgG positive plasma cells were seen and $45 \%$ plasma cells showed IgG4 positivity. Her serum IgG4 levels were elevated (142 mg/dL). A diagnosis of IgG4-RM was established based on Japanese consensus criteria and the patient was started on glucocorticoids therapy $(1 \mathrm{mg} / \mathrm{kg})$. She had improved pain and discharge in follow-up visit after two weeks. Methotrexate (15 mg/week) was added as steroid sparing agent. Her follow-up ultrasound of breast after two months revealed significant reduction in size of abscess $(50 \times 20 \mathrm{~mm} \rightarrow 24 \times 9 \mathrm{~mm})$ (Figure 2). After three months of treatment, the abscess resolved completely. Her oral steroids were gradually tapered and she was continued
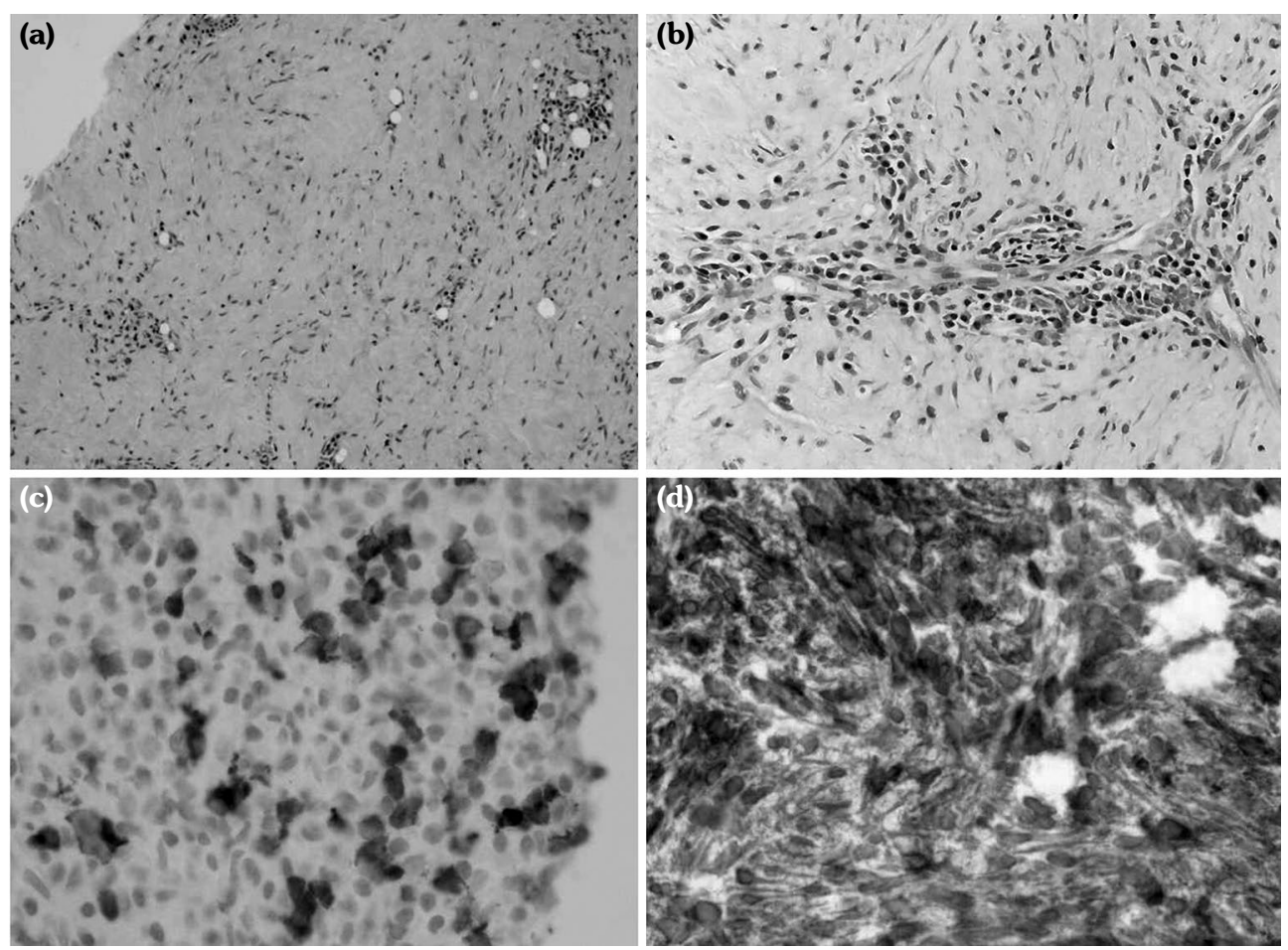

Figure 1. Histopathological examination showing (a) storiform fibrosis $(\mathrm{H}-\mathrm{E} \times 10)$,

(b) obliterative phlebitis $(\mathrm{H}-\mathrm{E} \times 40)$, (c) immunoglobulin $\mathrm{G}+$ plasma cells $(\mathrm{IHC} \times 40)$ and

(d) immunoglobulin $\mathrm{G} 4+$ plasma cells $(\mathrm{IHC} \times 40)$. 

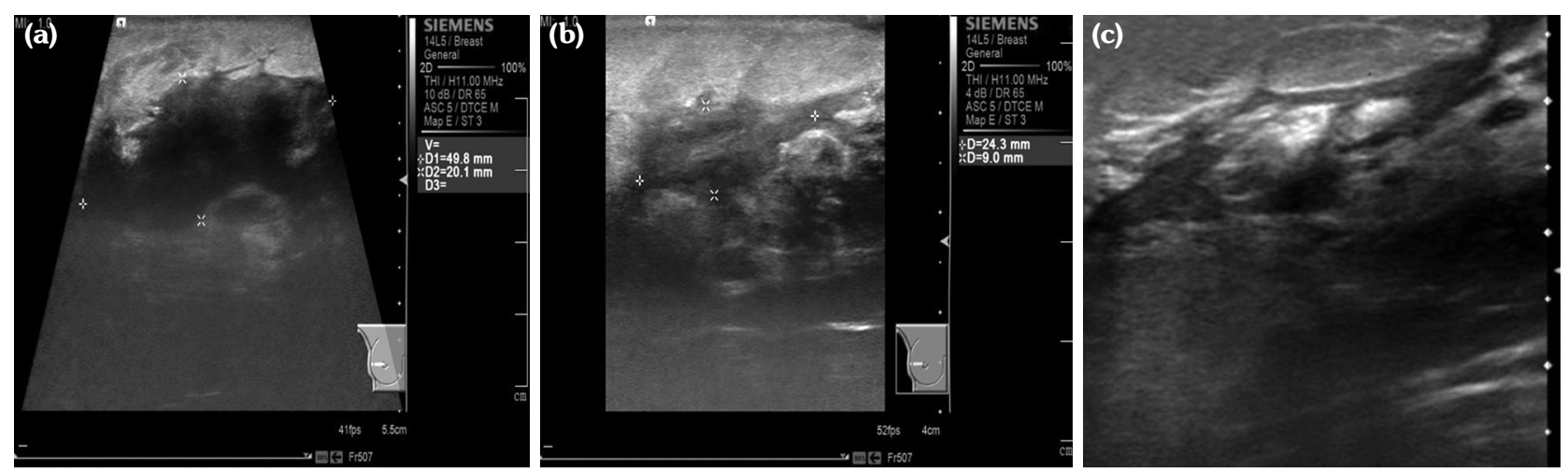

Figure 2. Ultrasonography of breast at (a) baseline, (b) after two months of treatment and (d) after four months of treatment.

on methotrexate therapy. At present, patient is symptom free at 12 months of regular follow-up and her IgG4 level is $85 \mathrm{mg} / \mathrm{dL}$. A written informed consent was obtained from the patient.

\section{DISCUSSION}

Immunoglobulin G4-related disease has been described in every organ system. Many medical conditions such as Mikulicz's syndrome, Küttner's tumor, autoimmune pancreatitis, retroperitoneal fibrosis and Riedel's thyroiditis which were thought to involve isolated organs only are now considered as part of the spectrum of IgG4-RD. ${ }^{1}$

Most of the epidemiological data of IgG4-RD is available from a Japanese cohort. ${ }^{4}$ It usually affects middle-aged individuals. Sex predominance depends upon the type of organ involved: autoimmune pancreatitis is more common in males while dacryoadenitis and sialadenitis is more common in females. ${ }^{5}$

No universal criteria for the diagnosis of IgG4-RD has been developed till date; however, consensus criteria proposed by Japanese IgG4 team, published in 2011, are commonly used. ${ }^{5}$ The major characteristics of this comprehensive diagnostic criteria are: serum IgG4 concentration $>135 \mathrm{mg} / \mathrm{dL}$, the infiltration of $>10 \mathrm{IgG} 4+$ cells per high-power field and an IgG4+/IgG+ cell ratio $>40 \%$. The cut-off of $135 \mathrm{mg} / \mathrm{dL}$ was based on receiver operating characteristic curves and its validity was confirmed in patients with AIP. ${ }^{6}$ Recent large cohort studies on IgG4-RD from the UK, Taiwan and Japan showed that serum IgG4 concentration $>135 \mathrm{mg} / \mathrm{dL}$ had overall sensitivities of $82.8 \%, 86 \%$ and $88 \%$, respectively, in diagnosing IgG4-RD.,8

In 2012, an international consensus on the pathology of IgG4-RD was published. For the diagnosis of IgG4-RD, two out of the following three criteria should be present: (i) dense lymphoplasmacytic infiltrate, (ii) storiform fibrosis and (iii) obliterative phlebitis. Neutrophilic infiltration and epitheloid cell granuloma argue against the presence of IgG4-RD. ${ }^{9}$

In the literature, IgG4-RM or IgG4related sclerosing disease of the breast has been described as "sclerosing mastitis" or "inflammatory pseudotumor". ${ }^{10,11}$ Because of its similarity with breast cancer, it is important to identify this entity. A total of 12 cases of IgG4-RM have been described in the literature till now. It commonly presents as painless palpable breast lump which is most commonly solitary, while multiple lesions have also been described. ${ }^{12}$ IgG4-RD presenting as an abscess is quite unusual and to the best of our knowledge, this happens to be the first case of IgG4$\mathrm{RD}$ presenting as breast abscess. Although the disease is known for tumefactive lesions, mechanism of its presentation as isolated abscess is unclear, while in the literature, lacrimal gland and hypophyseal abscesses have been described as a part of IgG4-RD spectrum but no case of IgG4-RM presenting as breast abscess has been described till now. ${ }^{13}$ At present, it is an orphan disease with novel clinical manifestations being reported across various medical specialities. 
In previously reported cases of IgG4-RM, more than half of the cases were treated with excision. ${ }^{12}$ In our case, the patient did not undergo any surgical intervention. She was treated with oral glucocorticoid and methotrexate. She is under regular follow-up and is doing well without any recurrence. On treatment front, rituximab is reserved for refractory cases or for those who have strong contraindications to steroid therapy although it needs further validation. ${ }^{14}$

\section{Declaration of conflicting interests}

The authors declared no conflicts of interest with respect to the authorship and/or publication of this article.

\section{Funding}

The authors received no financial support for the research and/or authorship of this article.

\section{REFERENCES}

1. Kamisawa T, Funata N, Hayashi Y, Eishi Y, Koike M, Tsuruta $\mathrm{K}$, et al. A new clinicopathological entity of IgG4-related autoimmune disease. J Gastroenterol 2003;38:982-4.

2. Hamano H, Kawa S, Horiuchi A, Unno H, Furuya N, Akamatsu T, et al. High serum IgG4 concentrations in patients with sclerosing pancreatitis. N Engl J Med 2001;344:732-8.

3. Saeki T, Saito A, Hiura T, Yamazaki H, Emura I, Ueno $\mathrm{M}$, et al. Lymphoplasmacytic infiltration of multiple organs with immunoreactivity for IgG4: IgG4-related systemic disease. Intern Med 2006;45:163-7.

4. Wallace ZS, Deshpande V, Mattoo H, Mahajan VS, Kulikova M, Pillai S, et al. IgG4-related disease: clinical and laboratory features in one hundred twenty-five patients. Arthritis Rheumatol 2015;67:2466-75.
5. Umehara H, Okazaki K, Masaki Y, Kawano M, Yamamoto M, Saeki $\mathrm{T}$, et al. Comprehensive diagnostic criteria for IgG4-related disease (IgG4-RD), 2011. Mod Rheumatol 2012;22:21-30.

6. Yu KH, Chan TM, Tsai $\mathrm{PH}$, Chen $\mathrm{CH}$, Chang PY. Diagnostic performance of serum IgG4 levels in patients with IgG4-related disease. Medicine (Baltimore) 2015;94:1707.

7. Inoue $\mathrm{D}$, Yoshida $\mathrm{K}$, Yoneda $\mathrm{N}$, Ozaki $\mathrm{K}$, Matsubara $\mathrm{T}$, Nagai $\mathrm{K}$, et al. IgG4-related disease: dataset of 235 consecutive patients. Medicine (Baltimore) 2015;94:680.

8. Culver EL, Sadler R, Simpson D, Cargill T, Makuch $\mathrm{M}$, Bateman AC, et al. Elevated serum IgG4 levels in diagnosis, treatment response, organ involvement, and relapse in a prospective IgG4-related disease UK cohort. Am J Gastroenterol 2016;111:733-43.

9. Deshpande V, Zen Y, Chan JK, Yi EE, Sato Y, Yoshino $\mathrm{T}$, et al. Consensus statement on the pathology of IgG4-related disease. Mod Pathol 2012;25:1181-92.

10. Cheuk W, Chan AC, Lam WL, Chow SM, Crowley $\mathrm{P}$, Lloydd R, et al. IgG4-related sclerosing mastitis: description of a new member of the IgG4related sclerosing diseases. Am J Surg Pathol 2009;33:1058-64.

11. Zen Y, Kasahara Y, Horita K, Miyayama S, Miura $S$, Kitagawa $S$, et al. Inflammatory pseudotumor of the breast in a patient with a high serum IgG4 level: histologic similarity to sclerosing pancreatitis. Am J Surg Pathol 2005;29:275-8.

12. Yokoe T, Hayashida T, Kikuchi M, Watanuki R, Nakashoji A, Maeda H, et al. IgG4-related mastopathy: A case report and literature review. Clin Case Rep 2018;6:1549-53.

13. Raab EL, Moayedpardazi HS, Naids SM, Friedman $\mathrm{AH}$, Meltzer MA. Lacrimal gland abscess in a child as a rare manifestation of IgG4-related disease. J AAPOS 2018;22:73-75.e1.

14. Khosroshahi A, Wallace ZS, Crowe JL, Akamizu $\mathrm{T}$, Azumi A, Carruthers $\mathrm{MN}$, et al. International consensus guidance statement on the management and treatment of IgG4-related disease. Arthritis Rheumatol 2015;67:1688-99. 